\title{
MACROSCOPIA DA MADEIRA DE EUCALYPTUS COMO FERRAMENTA PARA IDENTIFICAÇÃO A CAMPO
}

\author{
PAULA ZANATTA ${ }^{1}$, TALITA BALDIN²*, DEBORA DUARTE RIBES ${ }^{3}$, PATRÍCIA SOARES \\ BILHALVA DOS SANTOS ${ }^{4}$ e DARCI ALBERTO GATTO ${ }^{3}$
}

\begin{abstract}
Summary: Macroscopy of Eucalyptus wood as a field identification tool. The objective of the research was to perform a macroscopic anatomical study of the woods of six species of the genus Eucalyptus and to contribute to their identification at the field level. Eucalyptus acmenoides, E. bosistoana, E. deanei, E. longifolia, E. muelleriana e E. racemosa wood, not yet described anatomically, were analyzed. For this study, the basic specific mass was estimated and the samples were sanded and scarified with a scalpel, analyzed with a magnifying glass (10x magnification) and macroscopically described according to the norms of procedures in studies of wood anatomy, proposed by Coradin. The results showed similarities among the species regarding color, texture, grain, odor and grouping of the pores. The anatomical characters related to the pore distribution and the axial parenchyma are fundamental for macroscopic differentiation and recognition of these species.
\end{abstract}

Key words: basic density, eucalypt, macroscopic features, wood identification.

\begin{abstract}
Resumo: O objetivo da pesquisa foi realizar um estudo anatômico macroscópico das madeiras de seis espécies do gênero Eucalyptus e contribuir para identificação destas em nível de campo. Foram analisadas madeiras de E. acmenoides, E. bosistoana, E. deanei, E. longifolia, E. muelleriana e E. racemosa, ainda não descritas macroscopicamente. Para esse estudo, foi estimada a massa específica básica e então, as amostras foram lixadas e escarificadas com bisturi, analisadas com lupa conta fios (aumento 10x) e macroscopicamente descritas de acordo com as normas de procedimentos em estudos de anatomia da madeira, propostas por Coradin. Os resultados demonstraram semelhanças entre as espécies quanto à coloração, textura, grã, odor e agrupamento de dos poros. Contudo, os caracteres anatômicos relacionados à distribuição de poros e o parênquima axial foram fundamentais para a diferenciação macroscópica e reconhecimento destas espécies.
\end{abstract}

Palavras-chave: características macroscópicas, eucalipto, massa específica básica, identificação de madeira.

\section{INTRODUÇÃO}

A madeira é um material constituído por um conjunto de diferentes tipos de células, as quais apresentam funções específicas de sustentação, armazenamento e transporte de substâncias vitais para a sobrevivência do vegetal (Kalita et al., 2016). O conhecimento da sua constituição anatômica é um importante ramo da ciência, fundamental no emprego de qualquer espécie arbórea, visto a ampla diversidade existente.

Ao transformar o material em madeira serrada, as características morfológicas são eliminadas, tais como folhas, flores e frutos, o que dificulta a identificação botânica. Uma das formas mais confiáveis de identificar uma espécie é por meio

\footnotetext{
1 Universidade do Estado de Santa Catarina, Centro de Ciências Agroveterinárias, Departamento de Engenharia Florestal, Lages, SC, Brasil.

2 Universidade Federal de Minas Gerais, Departamento de Engenharia Florestal, Montes Claros, MG, Brasil.

${ }^{3}$ Universidade Federal de Pelotas, Pós-graduação em Ciência e Engenharia de Materiais, Pelotas, RS, Brasil.

${ }^{4}$ Universidade Federal do Pará, Departamento de Engenharia Florestal, Altamira, PA, Brasil.

*talita.baldin@hotmail.com
} 
da anatomia da madeira em nível microscópico aliada a chaves dicotômicas. Contudo, para que isso seja possível, são necessários equipamentos e técnicas mais avançadas, bem como profissionais qualificados, o que demanda mais custo e tempo para elaboração da técnica. Para que ocorra a identificação rapidamente em nível de campo, seja no recebimento no pátio ou como fiscalização de transporte de madeiras, a macroscopia, com visualização de caracteres anatômicos a olho nu ou com aumento máximo de $10 x$, pode atuar como uma ferramenta eficaz no reconhecimento de espécies, com um alto grau de confiabilidade (Zenid \& Ceccantini, 2012).

Entre as diferentes madeiras, as do gênero Eucalyptus, pelo elevado número de exemplares, são de difícil identificação por apresentarem semelhanças visuais, o que muitas vezes leva à utilização equivocada causada pela ausência do devido conhecimento da espécie e de suas propriedades intrínsecas. Sendo assim, a falta de informações e práticas inapropriadas (cor, textura, gosto e cheiro) executadas a campo levam a identificações errôneas, fraudes e emprego inadequado das espécies.

Atualmente as espécies do gênero Eucalyptus são as mais plantadas no mundo, com 20 milhões de hectares em cerca de 90 países, nas regiões da África, América, Ásia, Austrália e Europa (Myburg et al. 2014). Estas, juntamente com o Pinus sp., são consideradas as principais matérias-primas da indústria brasileira de processamento de toras, produção de polpa e celulose, com aplicações nos mais diversos segmentos de produção madeireira.

Existem mais de 700 espécies catalogadas de Eucalyptus, o que instiga os pesquisadores a realizar minuciosas avaliações, a fim de obter maiores informações sobre suas diferenças (Vital, 2007). Apesar da importância da madeira de eucalipto no setor industrial mundial, pesquisas relacionadas à caracterização anatômica macroscópica do gênero são pouco exploradas. As investigações mais abordadas são relacionadas com dimensões de fibras, as quais fornecem embasamento científico para indústria de papel e celulose. Contudo, pelas potenciais características do gênero, há um emprego expressivo na construção civil e como madeira serrada, os quais necessitam do conhecimento de suas características anatômicas para reconhecimento e destino final. Assim, com a identificação macroscópica, a partir das descrições anatômicas a olho nú ou com baixa magnificação (10x), é possível reconhecer e predizer o uso correto da madeira, o que evita gastos desnecessários e problemas futuros (Alves et al., 2012; Pirralho et al., 2014; Giachi et al., 2016).

Frente ao crescente uso de madeiras de Eucalyptus, oriundas de reflorestamento, surge a necessidade de descrever e comparar espécies desse gênero em decorrência da grande semelhança anatômica. Nesse sentido, o presente trabalho tem como objetivo realizar um estudo anatômico macroscópico de madeiras de seis espécies do gênero Eucalyptus (E. acmenoides, E. bosistoana, E. deanei, E. longifolia, E. muelleriana e E. racemosa), cultivadas no Rio Grande do Sul e contribuir para reconhecimento destas em nível de campo.

\section{Material e Métodos}

As madeiras estudadas foram coletadas com 43 anos de idade em plantios comerciais instalados no ano de 1974, de propriedade da empresa Celulose RioGrandense (CMPC), na cidade de Barra do Ribeiro, Rio Grande do Sul. O clima da região onde os povoamentos estão implantados caracterizase como subtropical úmido, predominando solos profundos, muito profundos e excessivamente bem drenados (CMPC, 2016).

Foram adquiridos discos no diâmetro a altura do peito (DAP), tomado a $1,30 \mathrm{~m}$ do nível do solo, de seis espécies de Eucalyptus: E. acmenoides, E. bosistoana, E. deanei, E. longifolia, E. muelleriana e E. racemosa. Junto aos discos de madeira coletouse material botânico, que serviu para a identificação das espécies. Todo material estudado foi coletado por Talita Baldin, na data de 11 de maio de 2015, no horto florestal Barba Negra da empresa CMPC Celulose Riograndense, na cidade de Barra do Ribeiro, Rio Grande do Sul, Brasil. As exsicatas, confeccionadas a partir do material coletado, foram incorporadas ao Herbário do Departamento de Ciências Florestais da Universidade Federal de Santa Maria (HDCF), sob os registros disponibilizados (Tabela 1).

Para a determinação da massa específica básica foram retiradas duas cunhas diametralmente oposta (Fig. 1), as quais permaneceram imersas em água até atingir o ponto de saturação necessário à medição. Os valores determinados para esta 


\section{P. Zanatta et al. - Macroscopia da madeira de Eucalyptus}

Tabela 1. Registro no herbário das espécies utilizadas no estudo.

\begin{tabular}{|lc|}
\hline \multicolumn{1}{|c}{ Espécie } & $\begin{array}{c}\text { Registro no herbário } \\
\text { (HDCF*) }\end{array}$ \\
\hline Eucalyptus acmenoides & 11.6762 \\
Eucalyptus bosistoana & 7.6758 \\
Eucalyptus deanei & 12.6763 \\
Eucalyptus longifolia & 13.6764 \\
Eucalyptus muelleriana & 5.6755 \\
Eucalyptus racemosa & 6.751 \\
\hline
\end{tabular}

*Herbário do Departamento de Ciências Florestais da Universidade Federal de Santa Maria (HDCF).

variável seguiram a metodologia proposta pela American Society for Testing and Materials D 239593 (ASTM, 1995).

Para análise descritiva dos caracteres gerais e anatômicos macroscópicos, foram utilizadas as normas de procedimentos em estudos de anatomia da madeira, propostas por Coradin (1991). Assim, nas cunhas restantes de cada disco, observaram-se: distinção entre cerne e alburno, cor, odor, grã, brilho, textura, camadas de crescimento e visibilidade, tipo, distribuição e frequência de poros, parênquima e raios.

O método empregado para preparar as amostras foi o de escarificação manual. Para isso, primeiramente, as amostras foram lixadas nos diferentes planos anatômicos da madeira (transversal, longitudinal tangencial e longitudinal radial), escarificadas manualmente com um bisturi, para melhor visualização das características, e, posteriormente escovadas para limpeza da superfície. No caso de avaliação de caracteres anatômicos, uma lupa conta fios (de fácil aquisição em campo) foi utilizada.

Para confirmar a análise descritiva realizada nas diferentes espécies, fez-se macrofotografias das seções transversal e tangencial de cada madeira. Estas foram obtidas por meio de uma câmera digital (Digital Still Camera DSC-S75 Sony) acoplada a uma lupa ótica Zeiss Stemi SV11. Os programas MI PRO standard v1.1 e GIMP 2 auxiliaram no processamento e manipulação das imagens geradas após o método de escarificação manual.

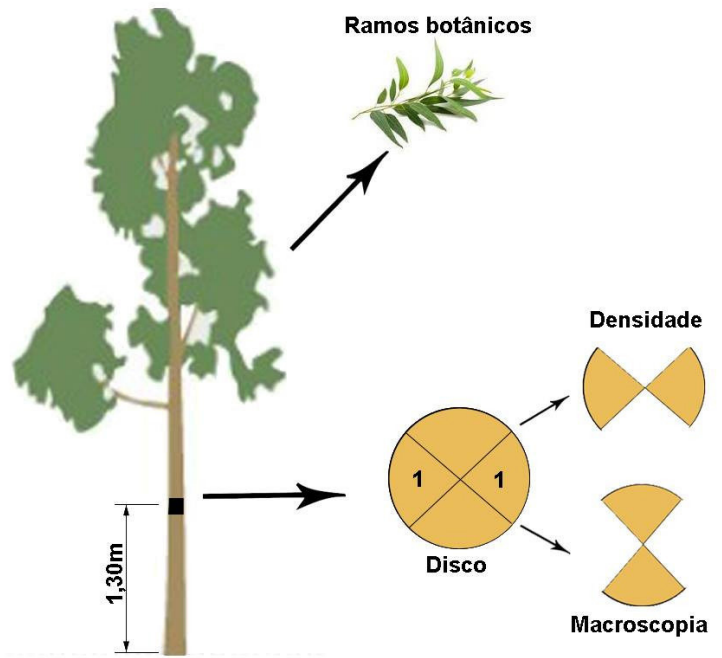

Fig. 1. Esquema de amostragem e localização das amostras para identificação botânica, massa específica e caracterização macroscópica.

\section{Resultados}

Eucalyptus acmenoides Schauer

a) Descrição dos caracteres gerais

A massa específica básica da madeira da espécie E. acmenoides é considerada média $\left(0,64 \mathrm{~g} \cdot \mathrm{cm}^{-3}\right)$. A cor da madeira definida como marrom muito pálida possuindo cerne e alburno indistintos. Apresenta brilho moderado na sua superfície e o odor é imperceptível. A grã é direita, textura é fina e as camadas de crescimento são distintas por uma zona com coloração mais escura.

\section{b) Descrição anatômica macroscópica}

No plano transversal, os poros são visíveis sob lente de 10x, com porosidade difusa uniforme, comumente solitários, aparentemente múltiplos, distribuídos em um ângulo de, aproximadamente, $45^{\circ}$ em relação aos raios (Fig. 2A). Os poros destacam-se em quantidade, classificados em muito numerosos de pequeno diâmetro. Parênquima axial é invisível e o radial apresenta raios finos e numerosos visíveis apenas sob lente 10x. No plano longitudinal tangencial o parênquima radial é visível sob lente 10x, não estratificado e normalmente com raios altos e largos (Fig. 2B). No plano radial o espelhado dos raios é pouco contrastado. A madeira apresenta camadas de crescimento individualizadas 
Bol. Soc. Argent. Bot. 53 (4) 2018
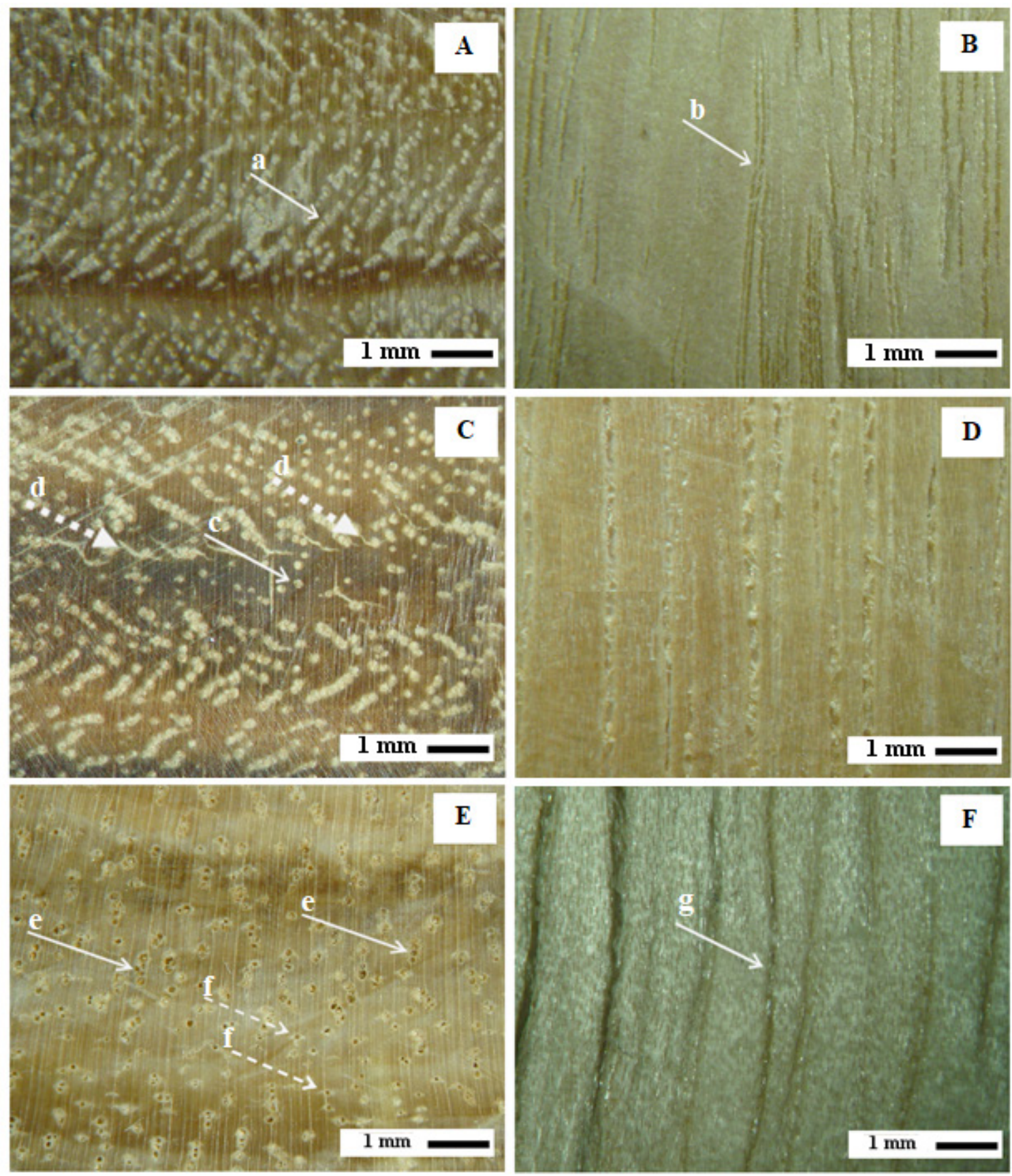

Fig. 2. A: Eucalyptus acmenoides em seção transversal evidenciando os poros distribuídos em um ângulo de aproximadamente $45^{\circ}$ em relação aos raios (seta a). B: Mesma espécie em plano longitudinal tangencial evidenciando raios altos (seta b). C :Eucalyptus bosistoana em seção transversal evidenciando os poros solitários (seta c) e parênquima axial, vasicêntrico e aliforme (seta d). D: Plano longitudinal tangencial em E. bosistoana. E: Seção transversal de Eucalyptus deanei evidenciando porosidade difusa, agrupamento radial, de dois a quatro poros, por vezes, solitários (seta e) e parênquima axial confluente, vasicêntrio e aliforme linear (seta f). F: Parênquima radial na mesma com raios altos, normalmente na mesma direção e altura (seta g), em plano longitudinal tangencial. 


\section{P. Zanatta et al. - Macroscopia da madeira de Eucalyptus}

por zonas fibrosas mais escuras, nestas, inclusive, os vasos tendem a ser sempre solitários e distribuídos de forma uniforme.

\section{Eucalyptus bosistoana F. Muell}

a) Descrição dos caracteres gerais

A madeira possui massa específica básica alta, $0,78 \mathrm{~g} \cdot \mathrm{cm}^{-3}$, coloração marrom claro, com cerne e alburno indistintos. A superfície é opaca e o odor imperceptível. A grã é direita, textura fina e as camadas de crescimento são distintas.

b) Descrição anatômica macroscópica

Os poros são visíveis sob lente $10 \mathrm{x}$ em plano transversal. A porosidade ocorre em anéis porosos, variando a sua frequência na camada de crescimento (Fig. 2C). Poros são solitários e aparentemente múltiplos, numerosos e diâmetro pequeno. O parênquima axial é vasicêntrico e aliforme linear, visível sob lente 10x. No plano longitudinal tangencial o parênquima radial é levemente visível a olho nu, não estratificado e normalmente com raios altos (Fig. 2D). No plano radial o espelhado dos raios não é contrastado. Madeira com camadas de crescimento individualizadas pela distribuição dos poros.

\section{Eucalyptus deanei Maiden}

a) Descrição dos caracteres gerais

Madeira de massa específica básica considerada média $\left(0,59 \mathrm{~g} \cdot \mathrm{cm}^{-3}\right)$. Coloração marrom muito pálida, possuindo cerne e alburno indistintos. Superfície brilhosa e odor imperceptível. A grã é classificada como ondulada, a textura é fina e as camadas de crescimento são pouco distintas.

\section{b) Descrição anatômica macroscópica}

Em plano transversal os poros são visíveis a olho nu e em porosidade difusa. Poros numerosos e de diâmetro médio, predominantemente em agrupamento radial, de dois a quatro, por vezes, solitários. Parênquima axial vasicêntrio e aliforme linear, este último, predominantemente em poros solitários (Fig. 2E). O parênquima radial é levemente visível a olho nu, finos e numerosos. No plano longitudinal tangencial o parênquima radial é visível a olho nu e com raios altos, normalmente na mesma direção e altura (Fig. 2F). Em plano radial o espelhado dos raios é pouco contrastado. A madeira de $E$. deanei apresenta camadas de crescimento individualizadas por zonas fibrosas mais escuras.
Eucalyptus longifolia Link

a) Descrição dos caracteres gerais

A madeira de E. longifolia tem massa específica básica média $\left(0,66 \mathrm{~g} \cdot \mathrm{cm}^{-3}\right)$. A cor, definida como marrom avermelhado no cerne e marrom claro na região do alburno. Apresenta brilho entre opaco e moderado na sua superfície e o odor é imperceptível. A grã é direita, a textura é fina e as camadas de crescimento são distintas apenas no alburno.

b) Descrição anatômica macroscópica

Em plano transversal os poros são visíveis sob lente $10 \mathrm{x}$, com porosidade em anéis porosos no alburno (Fig. 3A) e difusa não uniforme no cerne e (Fig 3B). Poros solitários, pouco numerosos a numerosos e de pequeno diâmetro. O parênquima axial é paratraqueal vasicêntrico, contudo no alburno é pouco visível sob lente 10x.

Em plano longitudinal tangencial o parênquima radial é visível apenas sob lente $10 x$, não estratificado e normalmente com raios altos. Em plano radial o espelhado dos raios não é contrastado. As camadas de crescimento são individualizadas por anéis porosos pela frequência dos poros na região do alburno.

\section{Eucalyptus muelleriana A.W. Howitt}

a) Descrição dos caracteres gerais

A massa específica básica da madeira de E. muelleriana é considerada alta $\left(0,72 \mathrm{~g} \cdot \mathrm{cm}^{-3}\right)$. A cor definida como marrom muito pálida, possuindo cerne e alburno indistintos. Apresenta brilho moderado na superfície e o odor é imperceptível. A grã é inclinada, a textura é média e as camadas de crescimento são pouco distintas.

\section{b) Descrição anatômica macroscópica}

Em plano transversal, os poros são visíveis sob lente $10 \mathrm{x}$ e a porosidade é em anel semicircular (Fig. 3C). Os poros são solitários, classificados como pouco numerosos e médios. Parênquima axial paratraqueal vasicêntrico e parênquima radial fino e numeroso, visíveis apenas sob lente 10x. No plano longitudinal tangencial o parênquima radial é visível a olho nu, não estratificado e normalmente com raios altos (Fig. 3D). Em plano radial o espelhado dos raios não é contrastado. Camadas de crescimento individualizadas por anéis semicirculares. 

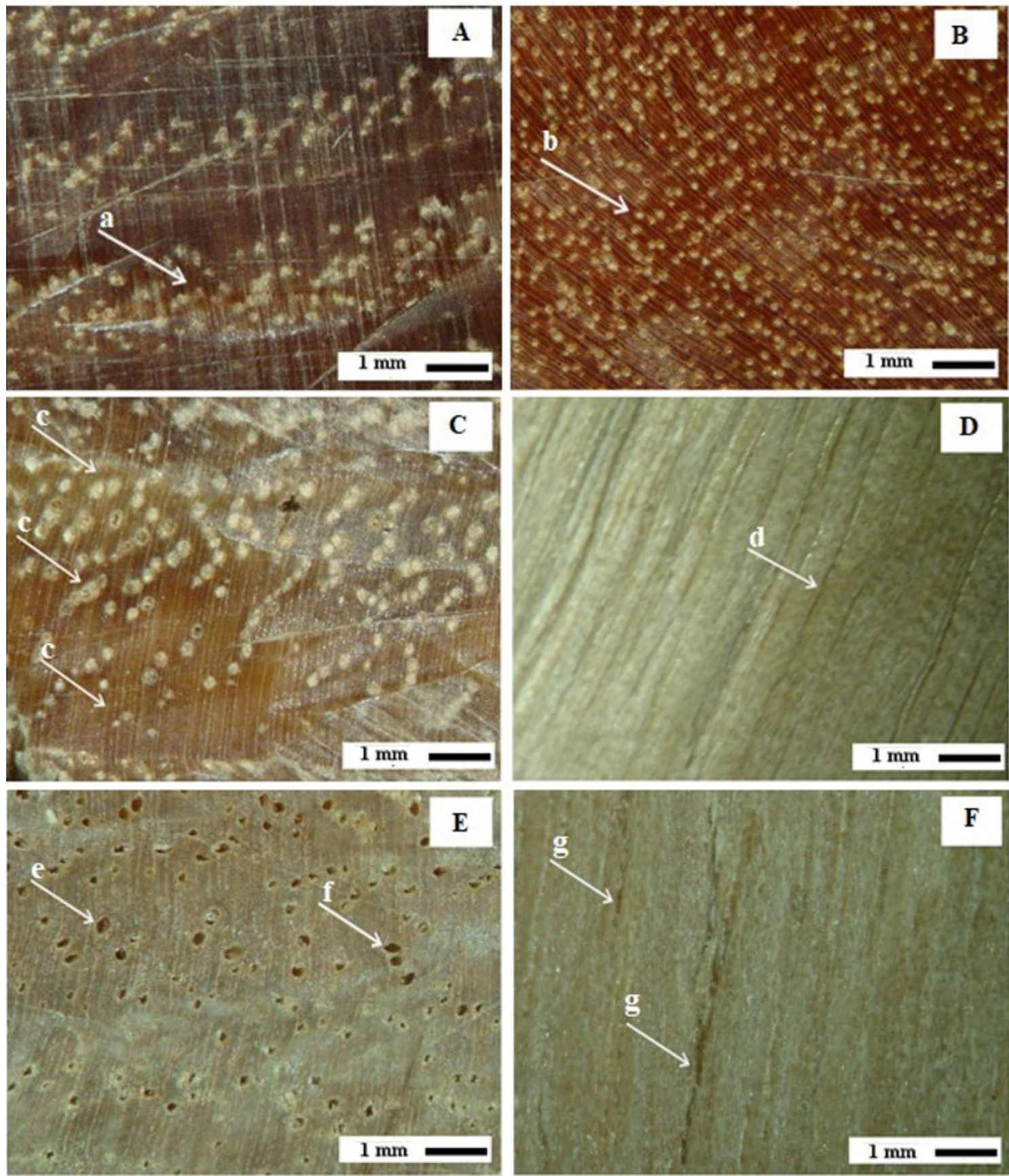

Fig. 3. A: Eucalyptus longifolia em seção transversal evidenciando porosidade em anel no alburno (seta a). B: Mesma espécie em seção transversal evidenciando a porosidade difusa do cerne (seta b). C: Seção transversal em E. muelleriana evidenciando porosidade semicircular e poros solitários (seta c). D: No plano longitudinal tangencial de E. muelleriana o parênquima radial (seta d). E: Eucalyptus racemosa em seção transversal evidenciando poros solitários (seta e) e agrupados (seta f). F: Parênquima radial (seta g) na seção tangencial de E. racemosa. 


\section{P. Zanatta et al. - Macroscopia da madeira de Eucalyptus}

Eucalyptus racemosa Cav.

a) Descrição dos caracteres gerais

Madeira com massa específica básica de 0,57 g. $\mathrm{cm}^{-3}$, classificada como de média. A cor da madeira é definida como marrom pálido na região do alburno e marrom levemente avermelhado no cerne. A superfície é brilhosa, com odor imperceptível. Grã inclinada, a textura é fina e as camadas de crescimento são distintas.

\section{b) Descrição anatômica macroscópica}

No plano transversal os poros são visíveis a olho nu, com porosidade em anel poroso (Fig. 3E). Poros solitários e em maior frequência na transição do anel de crescimento, atribuindo um aspecto de faixa em algumas regiões. São pouco numerosos e de diâmetro médio, com ampla variação de tamanho. $\mathrm{O}$ parênquima axial é praticamente invisível, contudo, nas regiões de grande aglomeração de poros, podem ser visualizados como vasicêntrico. O parênquima radial é visível sob lente 10x e pode ser classificado como fino e numeroso. No plano longitudinal tangencial o parênquima radial é visível a olho nu, não estratificado e normalmente com raios altos (Fig. 3F). Em plano radial o espelhado dos raios é bem contrastado. A madeira apresenta camadas de crescimento individualizadas por distribuição dos poros em anéis porosos, demarcados pela sua maior frequência e por variação de diâmetro.

\section{Discussão}

O método de escarificação manual para descrição de espécies é simples, rápido e utiliza ferramentas acessíveis. Acredita-se que em nível de campo, este se torna adequado para identificar, reconhecer e empregar corretamente madeiras (exóticas ou nativas; autorizadas ou restritas ao corte) que circulam no mercado, em um grau alto de confiabilidade. No entanto, por se tratar de uma técnica que utiliza um preparo superficial com lixas, os poros ficam obstruídos, o que limita a detecção de tilos nas estruturas porosas.

As descrições das características anatômicas macroscópicas e as fotomacrografias das diferentes espécies de Eucalyptus visualizadas anteriormente podem ser consideradas ferramentas importantes para serem utilizadas na identificação de madeiras em nível de campo. Estas dão um embasamento científico, considerado seguro e rápido na fiscalização de madeiras transportadas em rodovias e auxiliam o reconhecimento destas seis espécies, como por exemplo, em serrarias.

A homogeneidade dentro de uma mesma família já é constatada na literatura, principalmente em nível macroscópico. Assim, estudos que envolvem a descrição de vasos, parênquima e raios se torna importante para descobrir peculiaridades que diferenciam as espécies, dentro de um mesmo gênero (Reis, 2015).

A massa específica básica das madeiras de $E$. bosistoana e E. mulleriana foram superiores às demais espécies estudadas e se assemelham ao E. botryoides e E. tereticornis, com $0,72 \mathrm{~g} . \mathrm{cm}^{-3}$ e $0,76 \mathrm{~g} . \mathrm{cm}^{-3}$, respectivamente, encontrado por Ávila Delucis et al. (2014), que também circulam e são empregadas no mercado madeireiro da região.

De modo geral, no que se refere aos caracteres gerais, houve similaridade quanto à coloração, grã, textura e odor, corroborando com a literatura de várias espécies de Eucalyptus (Dadswell \& Burnell, 1932; Dadswell, 1972; Metcalfe \& Chalk, 1950; Van Vliet \& Baas, 1984; Détienne \& Jacquet, 1983; Pirralho et al., 2014).

Com relação à estrutura do lenho, unicamente o E. acmenoides demonstrou poros distribuídos uniformemente na madeira, e também parênquima invisível e camadas de crescimento distintas por zonas mais escuras. Estas características, juntamente com a coloração, são similares às encontradas por Alves et al. (2012) ao descrever o E. cloeziana, as quais podem ser facilmente confundidas.

O parênquima do tipo vasicêntrico e aliforme apareceram em abundância na maioria das espécies. Verificou-se que para o gênero Eucalyptus esta estrutura é comumente encontrada em espécies como: E. grandis, E. citriodora, E. pellita, E. globulus e E. gummifera (Tomazello Filho, 1985; Poubel et al., 2011; Alves et al., 2012). O agrupamento dos poros se destacou por ser em maior parte solitários, igualmente a $E$. grandis e E. urophylla (Brisola \& Demarco, 2011).

Acredita-se que caracteres como porosidade e tipo de parênquima podem fornecer informações valiosas para diferenciação e identificação de espécies do gênero. Assim, a Tabela 2, esboça as descrições consideradas mais relevantes e que facilitam o reconhecimento das madeiras estudadas. 
Bol. Soc. Argent. Bot. 53 (4) 2018

\begin{tabular}{|c|c|c|c|c|c|c|c|}
\hline $\begin{array}{l}0 \\
\frac{0}{\pi} \\
0 \\
0\end{array}$ & 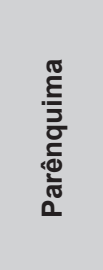 & 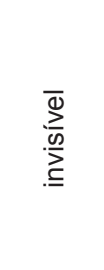 & 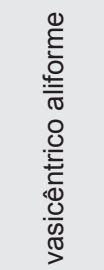 & 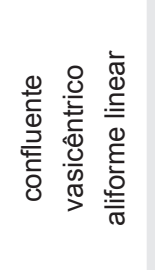 & 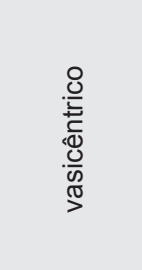 & 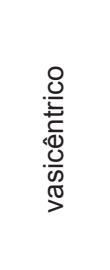 & 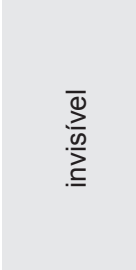 \\
\hline 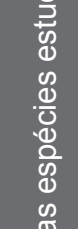 & $\begin{array}{l}\frac{0}{0} \\
\frac{\pi}{0} \\
\frac{0}{n} \\
0 \\
0 \\
0\end{array}$ & 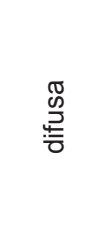 & $\begin{array}{l}0 \\
0 \\
0 \\
0 \\
\frac{0}{0} \\
\frac{0}{0} \\
\frac{0}{0} \\
\frac{0}{\pi} \\
\frac{\pi}{0}\end{array}$ & 忽 & 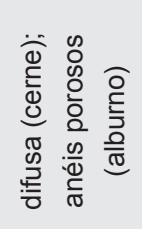 & 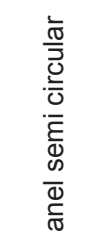 & $\begin{array}{l}0 \\
0 \\
0 \\
0 \\
\overline{0} \\
\frac{0}{0} \\
\frac{0}{01} \\
\frac{0}{\pi}\end{array}$ \\
\hline 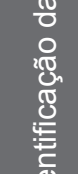 & : & 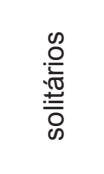 & 告 & 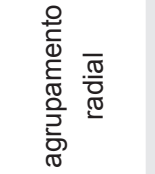 & 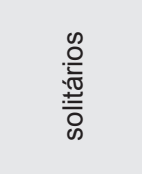 & 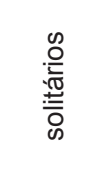 & 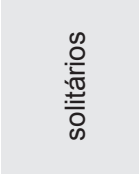 \\
\hline 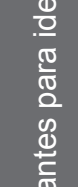 & 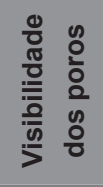 & $\begin{array}{l}\text { o } \\
0 \\
\Phi \\
\underline{ \pm} \\
\underline{\omega} \\
0 \\
0 \\
0\end{array}$ & 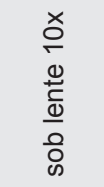 & $\begin{array}{l}\vec{\jmath} \\
\circ \\
\text { 응 }\end{array}$ & 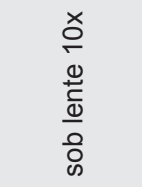 & 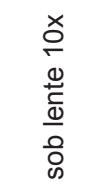 & $\begin{array}{l}\text { ว } \\
\circ \\
\text { 등 }\end{array}$ \\
\hline 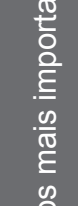 & 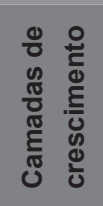 & 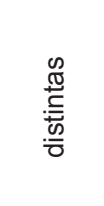 & 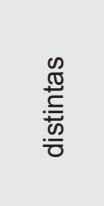 & 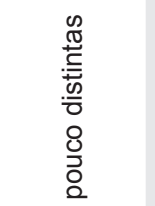 & 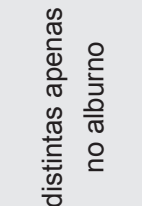 & 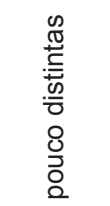 & 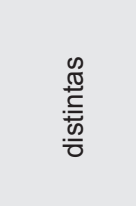 \\
\hline 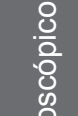 & 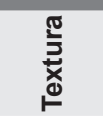 & 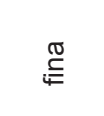 & $\underset{⿱ 乛}{\stackrel{D}{E}}$ & $\underset{⿱ 乛}{\stackrel{D}{\rightleftarrows}}$ & $\underset{\leftarrow}{\stackrel{D}{\rightleftarrows}}$ & $\begin{array}{l}\frac{\sigma}{\bar{\sigma}} \\
\stackrel{\oplus}{\varrho} \\
\varepsilon\end{array}$ & $\underset{\stackrel{\mathbb{J}}{\rightleftarrows}}{ }$ \\
\hline 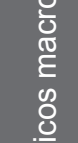 & 站 & $\begin{array}{l}\frac{0}{0} \\
\frac{\pi}{0} \\
\frac{0}{0} \\
\frac{0}{0} \\
\varepsilon\end{array}$ & $\begin{array}{l}\text { O্ } \\
\frac{\pi}{0}\end{array}$ & 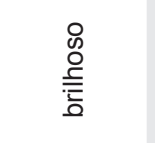 & 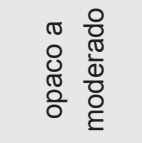 & $\begin{array}{l}\frac{0}{0} \\
\frac{\pi}{\frac{\pi}{\pi}} \\
\frac{0}{0} \\
\varepsilon\end{array}$ & 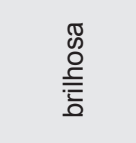 \\
\hline 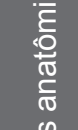 & 霡 & 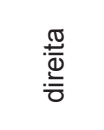 & 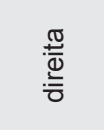 & $\begin{array}{l}\frac{\pi}{\frac{\pi}{0}} \\
\frac{\pi}{2} \\
\frac{\pi}{0} \\
0\end{array}$ & 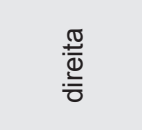 & 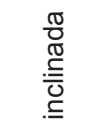 & 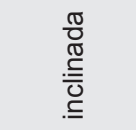 \\
\hline 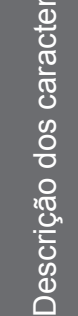 & 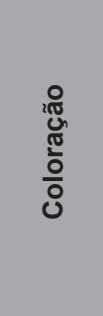 & 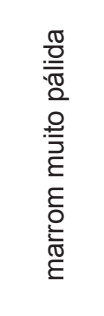 & $\begin{array}{l}\frac{0}{\frac{0}{\pi}} \\
\frac{0}{0} \\
\frac{0}{0} \\
\frac{0}{\overline{0}} \\
\varepsilon\end{array}$ & 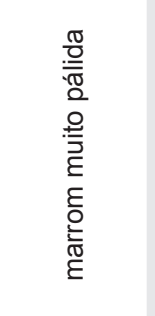 & 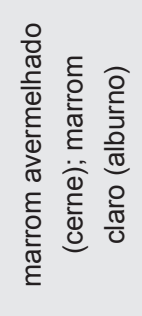 & 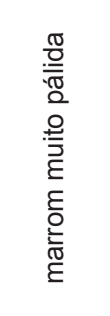 & 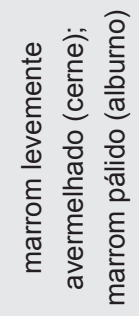 \\
\hline $\begin{array}{l}\frac{10}{0} \\
\frac{0}{10}\end{array}$ & $\begin{array}{l}\stackrel{m}{\tilde{E}} \\
\dot{0} \\
\dot{0}\end{array}$ & $\begin{array}{l}\Xi \\
0\end{array}$ & $\begin{array}{l}\infty \\
\stackrel{\infty}{0} \\
\stackrel{0}{0}\end{array}$ & 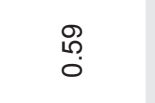 & $\begin{array}{l}\bullet \\
\stackrel{0}{0}\end{array}$ & $\begin{array}{l}\mathbb{N} \\
0 \\
0\end{array}$ & 卢 \\
\hline & 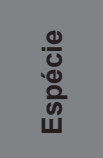 & 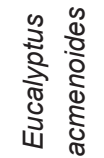 & 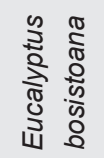 & 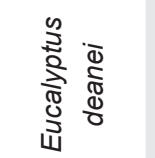 & 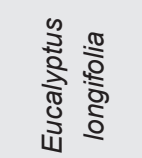 & 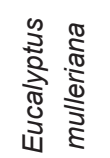 & 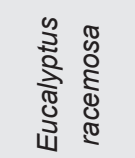 \\
\hline
\end{tabular}




\section{P. Zanatta et al. - Macroscopia da madeira de Eucalyptus}

\section{Conclusão}

As espécies do gênero Eucalyptus estudadas demonstraram semelhança nas caracterizações gerais e anatômicas. Contudo, uma riqueza de detalhes essenciais foi observada para contribuir na correta identificação e diferenciação das espécies estudadas. $\mathrm{O}$ método de escarificação com bisturi e análise com lupa conta fios (aumento $10 \mathrm{x}$ ) é rápido e eficiente para reconhecimento em nível de campo. Recomenda-se ao identificador atenção em detalhes anatômicos, quanto à distribuição de poros e camadas de crescimento, bem como parênquima axial.

Após o corte da árvore e exclusão de todos os caracteres botânicos, as descrições realizadas podem ser utilizadas como ferramentas de reconhecimento e distinção entre as espécies em nível de campo. Sendo assim, estudos anatômicos de fácil abordagem se tornam importantes e podem atuar no auxílio a órgãos de fiscalização e contribuir uma garantia na comercialização legal de madeiras.

\section{Agradecimentos}

Os autores agradecem a CMPC pela disponibilidade das madeiras de Eucalyptus e Conselho Nacional de Desenvolvimento Científico e Tecnológico [151833/2018-9].

\section{Bibliografia}

AMERICAN SOCIETY FOR TESTING AND MATERIALS - ASTM D 2395-93. 1995. Test methods for specific gravity of wood and wood-base materials. Philadelphia.

ALVES, R. C., J. T. S. OLIVEIRA., J. MOTTA. \& J. B. PAES. 2012. Caracterização anatômica macroscópica de madeiras folhosas comercializadas no Estado do Espírito Santo. Floresta e Ambiente 19: 352-361.

DE AVILA DELUCIS, D. A. GATTO, P. H. G. CADEMARTORI, A. L. MISSIO \& E. SCHNEID. 2014. Propriedades físicas da madeira termorretificada de quatro folhosas. Floresta e Ambiente. 21: 99-107.

BRISOLA, S. H. \& D. DEMARCO. 2011. Análise anatômica do caule de Eucalyptus grandis, E. urophylla e E. grandis x urophylla: desenvolvimento da madeira e sua importância para a indústria. Scientia Forestalis. 39: 317-330.

CMPC, CELULOSE RIOGRANDENSE. 2016. Plano de Manejo 2016: Resumo Público [online]. Disponível em: http://www.celuloseriograndense.com.br/docs/ dl/plano_de_manejo_web.pdf [Acesso em 10 Abril 2017].

CORADIN, V. T. R. \& G. B. MUNIZ. 1991. Normas de procedimentos em estudos de anatomia de madeira; 1 : Angiosperma; 2: Gimnospermae. Brasilia: IBAMA. DIRPED, Laboratorio de Produtos Florestais.

DADSWELL, H. E. The anatomy of the Eucalyptus woods. 1972. Division of Applied Chemistry Technological Paper 66: 1-28.

DADSWELL, H. E. \& M. BURNELL. 1932. Methods for the identification of the coloured woods of the genus Eucalyptus. CSIR Div. For. Prod., Technical Paper No. 5.

DÉTIENNE, P. \& P. JACQUET. 1983. Atlas d'identification des bois de l'Amazonie et des régions voisines. Nogentsur-Marne: Gerdat-ctft.

GIACHI, G., M. C., GUIDOTTI, S. LAZZERI, L. SOZZI \& N. MACCHIONI. 2016. Wood identification of the headrests from the collection of the Egyptian Museum in Florence. Journal of Archaeological Science: Reports 9: 340-346.

KALITA, K., N. DAS, P. K. BORUAH \& U. SARMA. 2016. Development of a strain measurement system for the study of effect of relative humidity on wood. Measurement 94: 265-272.

METCALFE, C. R. \& L. CHALK. 1950. Anatomy of the Dicotyledons. Clarendon Press: Oxford.

MYBURG, A. A., D. GRATTAPAGLIA, G. A. TUSKAN, U. HELLSTEN, R. D. HAYES, J. GRIMWOOD \& D. M. GOODSTEIN. 2014. The genome of Eucalyptus grandis. Nature 510: 356-362.

PIRRALHO, M., D. FLORES, V. B. SOUSA, T. QUILHÓ, S. KNAPICA \& H. PEREIRA. 2014. Evaluation on paper making potential of nine Eucalyptus species based on wood anatomical features. Ind. Crop. Prod. 54: 327334.

POUBEL, D., R. A. GARCIA, J. V. F. LATORRACA \& A. M. CARVALHO. 2011. Estrutura anatômica e propriedades fisicas da madeira de Eucalyptus pellita $\mathrm{F}$. Muell. Floresta e Ambiente 18: 117-126.

REIS, A. Anatomia da madeira de quatro espécies de Aspidosperma Mart. \& Zucc. 2015. Comercializadas no estado do Pará, Brasil. Revista Ciência da Madeira 6: 47-62.

TOMAZELLO FILHO, M. 1985. Estrutura anatômica da madeira de oito espécies de eucalipto cultivadas no Brasil. Scientia Forestalis 29: 5-36.

VAN VLIET, G. J. C. M. P. \& BAAS. 1984. Wood anatomy and classification of the Myrtales. Ann. Mo. Bot. Gard. 71: 783-800.

VITAL, M. H. F. 2007. Impacto ambiental de florestas de eucalipto. Revista do BNDES 14: 235-276.

ZENID, G. J. \& G. C. T. CECCANTINI. 2007. Derivados, Produtos. Identificação macroscópica de madeiras. São Paulo: IPT.

Recibido el 3 de julio de 2018, aceptado el 17 de septiembre de 2018. Editora: Ana M. Gonzalez. 
